\title{
Forest Fire Evolution Prediction Using a Hybrid Intelligent System
}

\author{
Aitor Mata ${ }^{1}$, Bruno Baruque ${ }^{2}$, Belén Pérez-Lancho ${ }^{1}$ Emilio Corchado ${ }^{1}$, \\ and Juan M. Corchado ${ }^{1}$ \\ ${ }^{1}$ Department of Computing Science and Automation, University of Salamanca, Spain \\ \{aitor, lancho, escorchado, corchado\} @usal.es \\ ${ }^{2}$ Department of Civil Engineering, University of Burgos, Spain \\ bbaruque@ubu.es
}

\begin{abstract}
Forest fires represent a quite complex environment and an accurate prediction of the fires generated is crucial when trying to react quickly and effectively in such a critical situation. In this study, an hybrid system is applied to predict the evolution of forest fires. The Case-Based Reasoning methodology combined with a summarization of SOM ensembles algorithm has been used to face this problem. The CBR methodology is used as the solution generator in the system, reusing past solutions given to past problems to generate new solutions to new problems by adapting those past solutions to the new situations to face. On the other hand, a new summarization algorithm (WeVoS-SOM) is used to organize the stored information to make it easier to retrieve the most useful information from the case base. The developed system has been checked with forest fires historical and experimental data. The WeVoS-CBR system presented here has successfully predicted the evolution of the forest fires in terms of probability of finding fires in a certain area.
\end{abstract}

\section{Introduction}

A hybrid artificial intelligence system [1], [2] is presented in this study. It is applied to generate predictions about the evolution of forest fires. That kind of systems combines symbolic and sub-symbolic techniques to construct more robust and reliable problem solving models. In this study a system based on Case-Based Reasoning (CBR) [3] and Topology Preserving Models [4] is presented and successfully applied.

Case-Based Reasoning systems have the potential to use past information in order to generate useful knowledge that may be used to solve new problems. Those systems should organise the information handled in order to improve the way that information is used. When the amount of information stored in a CBR system grows, the results obtained are normally better. But the growth of the case base (internal structure where the data is accumulated) also implies a more difficult retrieval process, where more information has to be considered in order to obtain the best possible collection of data.

The summarization algorithm presented here, WeVoS-SOM (Weighted Voting Summarization of SOM ensembles) [5] represents the organizing system of the internal structure of the data in the CBR system. With such an inner organization, it is 
easier to locate the new data that is introduced in the system and to retrieve the needed information to solve new problems.

The combination of both, the generalization power of the CBR methodology and the organizational capabilities of the WeVoS-SOM algorithm generates a system that has been used to generate predictions in a natural environment such as forest fires. Historical data have been used to check the correction of the system, where the predictions generated were compared with the actual past data.

This paper is organized as follows. In the second section the Case-Based Reasoning methodology is introduced and briefly explained. In the third section, the novel summarization algorithm presented in this paper is explained and analyzed. Next, WeVOS-CBR, the developed system is described, and its main phases are detailed. Finally, some results of the application of the system to the forest fire case study and some conclusions are shown.

\section{Introducing the CBR Methodology}

Case-Based Reasoning [3] origins are in knowledge based systems. CBR systems solve new problems acquiring the needed knowledge from previous situations [6]. The main element of a CBR system is the case base, a structure that stores the information used to generate new solutions.

The learning capabilities of CBR systems are due to its own structure, composed of four main stages [7]: retrieve, reuse, revision and retain. The first stage is called retrieve, and consists in finding the cases (from the case base) that are most similar to the new problem. Once a set of cases is extracted from the case base, they are reused by the system. In this second stage (reuse), the selected cases are adapted to fit in the new problem. After applying the new solution to the problem, that solution is revised to check its performance. If it is an acceptable solution, then it is retained by the system and could eventually serve as a solution to future problems.

As a methodology [3], CBR has been used to solve a great variety of problems [8],[9]. It is a cognitive structure that can be easily applied to solve problems such as those related with soft computing, since the procedures used by CBR are quite easy to assimilate by soft computing approaches. CBR has also helped to create applications related to quite different environments. Different kinds of neural networks such as ART-Kohonen [10] or Growing Cell Structures [11] have been combined with CBR to automatically create the inner structure of the case base. Some effort has also been devoted to the case-based maintenance issue [12].

It is easy to understand that the case base is one of the key elements of a CBR system. It is crucial to dispose of a great amount of data, but properly organized. The quantity is important, but if there is no order within the stored cases, it could become impossible to obtain all the knowledge that such an accumulation of data may offer. This is why an algorithm like the WeVoS-SOM that is going to be explained next, is so useful to CBR systems.

\section{Explaining the WeVoS-SOM Algorithm}

Case-Based Reasoning systems are highly dependent on stored information. The new algorithm presented here, Weighted Voting Summarization of SOM ensembles 
(WeVoS-SOM) (Baruque et al.) is used to organize the data that is accumulated in the case base. It is also used to recover the most similar cases to the proposed problem.

The main objective of the new fusion of an ensemble of topology preserving maps [4] algorithm presented here, WeVoS-SOM, is to generate a final map processed unit by unit. Instead of trying to obtain the best position for the units of a single map trained over a single dataset, it aims to generate several maps over different parts of the dataset. Then, it obtains a final summarized map by calculating by consensus which is the best set of characteristics vector for each unit position in the map. To do this calculation, first this meta-algorithm must obtain the "quality" [13] of every unit that composes each map, so that it can relay in some kind of informed resolution for the fusion of neurons.

The final map obtained is generated unit by unit. The units of the final map are first initialized by determining their centroids in the same position of the map grid in each of the trained maps. Afterwards, the final position of that unit is recalculated using data related with the unit in that same position in every of the maps of the ensemble. For each unit, a sort of voting process is carried out as shown in Eq. 1:

$$
V(p, m)=\frac{\left|x_{p, m}\right|}{\sum_{1}^{M}\left|x_{p}\right|} \cdot \frac{q_{p, m}}{\sum_{1}^{M} q_{p}}
$$

where, $V p, m$ is the weight of the vote for the unit included in map $m$ of the ensemble, in its position $p ; M$ is the total number of maps in the ensemble; $x p, m$ is the binary vector used for marking the dataset entries recognized by the unit in position $p$ of map $m$; and, $q p, m$ is the value of the desired quality measure for the unit in position $p$ of map $m$.

The final map is fed with the weights of the units as it is done with data inputs during the training phase of a SOM, considering the "homologous" unit in the final map as the BMU. The weights of the final unit will be updated towards the weights of the composing unit. The difference of the updating performed for each "homologous" unit in the composing maps depends on the quality measure calculated for each unit. The higher quality (or the lowest error) of the unit of the composing map, the stronger the unit of the summary map will be updated towards the weights of that neuron. The summarization algorithm will consider the weights of a composing unit "more suitable" to be the weights of the unit in the final map according to both the number of inputs recognized and the quality of adaptation of the unit (Eq. 1). With this new approach it is expected to obtain more faithful maps to the inner structure of the dataset.

This algorithm generates a structure where similar data is placed close together, with a clear relationship between the distribution of the initial data and the structure obtained by the algorithm. This relation between reality and inner structure is quite useful to a CBR system, where the stored information must be used in future situations to obtain future solutions.

Next, the system developed combining the CBR methodology and the WeVoSSOM algorithm is explained, focusing on the main phases of the CBR cycle.

\section{An Hybrid Forecasting System}

CBR have already been used to generate predictions in complicated environments where different parameters were involved [14]. In this occasion, the CBR methodology 
is used in combination with a summarization of SOM ensembles algorithm, in order to improve its results. The WeVos-CBR system presented here is able to generate predictions using past information as a source of knowledge to solve new problems. The available information is divided into cases that are stored in the case base. Those cases are structured, using the WeVoS-SOM algorithm.

When a new problem should be solved by the system, then the most similar data to the problem is retrieved from the case base. Then, the inner organization generated by the WeVoS-SOM algorithm is useful to recover those elements more similar to the one that is introduced in the system as a problem. The retrieved cases are used to generate the new solution, by feeding a neural network, trained to generate solutions. If the solution generated is good enough to be proposed to the user, then it is also stored in the case base, to serve as new data to solve new problems. All this process, covering the four main phases of the CBR cycle explained before, is covered by the next sub-sections.

\subsection{Creating the Case Base and Retrieving the Most Similar Cases}

When the case base is created the WeVoS-SOM algorithm is used to structure it. The graphical capabilities of this novel algorithm are used in this occasion to create a model that represents the actual variability of the parameters stored in the cases. At the same time, the inner structure of the case base will make it easier to recover the most similar cases to the problem cases introduced in the system.

The WeVoS-SOM algorithm is also used to recover the most similar cases to the problem introduced in the system. The case base is organized by using the WeVoS-SOM algorithm. This organization keeps similar values stored close one to another. When a new problem comes into the system, then its virtual position into the inner structure of the case base is calculated. The system tries to store the problem into the case base like if it was a solution. That virtual allocation serves to calculate the position of the problem into the case base, and to recover those elements that are located close to that virtual position. Those retrieved cases are used in the next stage to generate the solution.

\subsection{Adapting the Retrieved Cases to Generate a New Solution}

After recovering the most similar cases to the problem from the case base, those cases are used to obtain a solution. Growing RBF networks [15] are used to generate the predicted solution corresponding to the proposed problem. The selected cases are used to train the GRBF network. This adaptation of the RBF network lets the system grow during the training phase in a gradual way increasing the number of elements (prototypes) which work as the centres of the radial basis functions. The error definition for every pattern is shown below:

$$
e=l / n \sum_{k=1}^{p}\left\|t_{i k}-y_{i k}\right\|
$$

where $t_{i k}$ is the desired value of the $k^{\text {th }}$ output unit of the $i^{\text {th }}$ training pattern, $y_{i k}$ the actual values of the $k^{\text {th }}$ output unit of the $i^{\text {th }}$ training pattern. After the creation of the GRBF network, it is used to generate the solution to the introduced problem. The solution will be the output of the network using as input data the retrieved cases. 


\subsection{Revising and Retaining the Proposed Solution}

In order to verify the precision of the proposed solution, Explanations are used [16]. To justify and validate the given solution, the retrieved cases are used once again. The selected cases have their own future associated situation. Considering the case and its solution as two vectors, a distance between them can be measured by calculating the evolution of the situation in the considered conditions. If the distance between the proposed problem and the solution given is smaller than the distances obtained from the selected cases, then the proposed solution is considered as a good one.

Once the proposed prediction is accepted, it can be stored in the case base in order to serve to solve new problems. It will be used equally than the historical data previously stored in the case base. The WeVoS-SOM algorithm is used again to introduce new elements in the case base.

\section{Case Study and Results}

Forest fires are a very serious hazard that, every year, cause significant damage around the world from an ecological, social, economical and human point of view [17]. These hazards are particularly dangerous when meteorological conditions are extreme with dry and hot seasons or strong wind. For example, fire is a recurrent factor in Mediterranean areas.

Table 1. Variables that define a case in the WeVoS-CBR system

\begin{tabular}{|c|c|c|}
\hline Variable & Definition & Unit \\
\hline Longitude & Geographical longitude & Degree \\
\hline Latitude & Geographical latitude & Degree \\
\hline Date & Day, month and year of the analysis & $\mathrm{dd} / \mathrm{mm} /$ уyуy \\
\hline Temperature & Celsius temperature in the area & ${ }^{\circ} \mathrm{C}$ \\
\hline Bottom pressure & Atmospheric pressure in the area & Newton $/ \mathrm{m}^{2}$ \\
\hline Area of the fires & $\begin{array}{l}\text { Surface covered by the fires present in the } \\
\text { analyzed area }\end{array}$ & $\mathrm{Km}^{2}$ \\
\hline Meridional Wind & Meridional component of the wind & $\mathrm{m} / \mathrm{s}$ \\
\hline Zonal Wind & Zonal component of the wind & $\mathrm{m} / \mathrm{s}$ \\
\hline Wind Strength & Wind strength & $\mathrm{m} / \mathrm{s}$ \\
\hline
\end{tabular}

Fires represent a complex environment, where multiple parameters are involved. In this sub-section, a series of applications and possible solutions are explained. There are different approaches to the forest fire problems [18], [19], [20] and [21], including all the main phases existing in the evolution of this kind of problem.

The WeVoS-CBR system presented here has been applied in this study to generate predictions in a forest fire scenario. Forest fires represent a great environmental risk. The main approaches that have been used to solve this problem come first from the detection of the fires [18], where different techniques have been applied. Once the fire is detected, it is important to generate predictions that should help to take decision in those contingency response situations [22]. 
The data used to check the WeVoS-CBR system was a subset of the available data that has not been previously used in the training phase. The predicted situation was contrasted with the actual future situation as it was known (historical data was used to train the system and also to test its correction). The parameters taken into account to create the cases stores in the system are listed in table 1. The proposed prediction was, in most of the variables, close to $90 \%$ of accuracy. To create the cases, the geographical area analyzed was divided into small squares, each of which were considered a case, with all its associated parameters (longitude, latitude, wind, pressure, temperature, etc.). The squares determine the area to be considered in every case. The problem is represented by the current situation of the area (all its parameters and the presence or not of fire). The solution is represented by the situation in that area in a future moment (same location but parameters changed to next day, or next step -if less than a day is considered in every step-).

In table 2 a summary of the results obtained is shown. In this table different techniques are compared. The table shows the evolution of the results along with the increase of the number of cases stored in the case base. All the techniques analyzed improve its results when increasing the number of cases stored. Having more cases in the case base, makes easier to find similar cases to the proposed problem and then, the solution can be more accurate.

The " $R B F$ " column represents a simple Radial Basis Function Network that is trained with all the data available. The network gives an output that is considered a solution to the problem. The " $C B R$ " column represents a pure CBR system, with no other techniques included, the cases are stored in the case bases and recovered considering the Euclidean distance. The most similar cases are selected and after applying a weighted mean depending on the similarity, a solution is proposed. The " $R B F+$ $C B R$ " column corresponds to the possibility of using a RBF system combined with CBR. The recovery from the CBR is done by the Manhattan distance and the RBF network works in the reuse phase, adapting the selected cases to obtain the new solution. The results of the " $R B F+C B R$ " column are, normally, better than those of the " $C B R$ ", mainly because of the elimination of useless data to generate the solution. Finally, the "WeVoS-CBR" column shows the results obtained by the proposed system, obtaining better results than the three previous analyzed solutions.

Table 2. Percentage of good predictions obtained with different techniques

\begin{tabular}{|l|l|l|l|l|}
\hline $\begin{array}{l}\text { Number of } \\
\text { cases }\end{array}$ & RBF & CBR & RBF + CBR & WeVoS-CBR \\
\hline 100 & $36 \%$ & $38 \%$ & $41 \%$ & $44 \%$ \\
\hline 500 & $40 \%$ & $44 \%$ & $47 \%$ & $51 \%$ \\
\hline 1000 & $48 \%$ & $51 \%$ & $59 \%$ & $65 \%$ \\
\hline 2000 & $52 \%$ & $58 \%$ & $66 \%$ & $73 \%$ \\
\hline 3000 & $56 \%$ & $62 \%$ & $70 \%$ & $78 \%$ \\
\hline 4000 & $61 \%$ & $66 \%$ & $73 \%$ & $85 \%$ \\
\hline 5000 & $66 \%$ & $70 \%$ & $78 \%$ & $90 \%$ \\
\hline
\end{tabular}




\section{Conclusions}

The comparison of the WeVoS-CBR system with other techniques and systems has shown its advantage in terms of efficiency and quality of the provided results. The performance of the WeVoS-CBR system was also compared with a previous local version of the system, with the same artificial intelligence techniques implemented, but without the use of agents and services. The tests performed consisted of the execution of the same series of predictions in both systems. There were 50 different prediction requests to be generated by the system. It was shown that, the current system was faster than previous versions (a 70\% faster in answering 50 successive requests)and the number of crashes produced when the load of work was increased, was quite lower (80\% less crashes when using the current system).

With the optimistic results obtained after applying the presented system to the forest fires scenario, a future line of investigation will be the application of this system to other complex environments where its predicting and generalizing capabilities could be successfully applied.

\section{Acknowledgments}

This research has been partially supported through projects of the Junta of Castilla and León BU006A08; project of the Spanish Ministry of Education and Innovation CIT-020000-2008-2 and CIT-020000-2009-12. The authors would also like to thank the vehicle interior manufacturer, Grupo Antolin Ingenieria S.A., within the framework of the project MAGNO2008 - 1028.- CENIT Project funded by the Spanish Ministry.

\section{References}

1. Abraham, A., Corchado, E., Corchado, J.M.: Hybrid learning machines. Neurocomputing 72, 2729-2730 (2009)

2. Herrero, Á., Corchado, E., Pellicer, M.A., Abraham, A.: MOVIH-IDS: A mobilevisualization hybrid intrusion detection system. Neurocomputing 72, 2775-2784 (2009)

3. Watson, I.: Case-based reasoning is a methodology not a technology. Knowledge-Based Systems 12, 303-308 (1999)

4. Kohonen, T.: The self-organizing map. Neurocomputing 21, 1-6 (1998)

5. Baruque, B., Corchado, E.: A weighted voting summarization of SOM ensembles. Data Mining and Knowledge Discovery, 1-29

6. Aamodt, A.: A Knowledge-Intensive, Integrated Approach to Problem Solving and Sustained Learning. In: Knowledge Engineering and Image Processing Group. University of Trondheim (1991)

7. Aamodt, A., Plaza, E.: Case-Based Reasoning: Foundational Issues, Methodological Variations, and System Approaches. AI Communications 7, 39-59 (1994)

8. Baruque, B., Corchado, E., Mata, A., Corchado, J.M.: A forecasting solution to the oil spill problem based on a hybrid intelligent system. Information Sciences 180, 2029-2043 (2010) 
9. Mata, A., Corchado, J.M.: Forecasting the probability of finding oil slicks using a CBR system. Expert Systems With Applications 36, 8239-8246 (2009)

10. Yang, B.S., Han, T., Kim, Y.S.: Integration of ART-Kohonen neural network and casebased reasoning for intelligent fault diagnosis. Expert Systems With Applications 26, 387395 (2004)

11. Diaz, F., Fdez-Riverola, F., Corchado, J.M.: Gene-CBR: A case-based reasoning tool for cancer diagnosis using microarray data sets. Computational Intelligence 22, 254-268 (2006)

12. Liu, C.-H., Chen, L.-S., Hsu, C.-C.: An Association-based Case Reduction Technique for Case-based Reasoning. Information Sciences 178, 3347-3355 (2008)

13. Pölzlbauer, G.: Survey and Comparison of Quality Measures for Self-Organizing Maps. In: Rauber, J.P. (ed.) Fifth Workshop on Data Analysis (WDA 2004). Elfa Academic Press, London (2004)

14. Corchado, J.M., Fdez-Riverola, F.: FSfRT: Forecasting System for Red Tides. Applied Intelligence 21, 251-264 (2004)

15. Karayiannis, N.B., Mi, G.W.: Growing radial basis neural networks: merging supervised andunsupervised learning with network growth techniques. IEEE Transactions on Neural Networks 8, 1492-1506 (1997)

16. Sørmo, F., Cassens, J., Aamodt, A.: Explanation in Case-Based Reasoning-Perspectives and Goals. Artificial Intelligence Review 24, 109-143 (2005)

17. Long, D.G.: Mapping fire regimes across time and space: Understanding coarse and finescale fire patterns. International Journal of Wildland Fire 10, 329-342 (2001)

18. Mazzeo, G., Marchese, F., Filizzola, C., Pergola, N., Tramutoli, V.: A Multi-temporal Robust Satellite Technique (RST) for Forest Fire Detection. Analysis of Multi-temporal Remote Sensing Images (2007)

19. Arrue, B.C., Ollero, A., Matinez De Dios, J.R.: An intelligent system for false alarm reduction in infrared forest-fire detection. Intelligent Systems and Their Applications 15, 64-73 (2000)

20. Muñoz, C., Acevedo, P., Salvo, S., Fagalde, G., Vargas, F.: Forest fire detection using NOAA/16-LAC satellite images in the Araucanía Region. Chile. Bosque 28, 119-128 (2007)

21. Rodríguez, R., Cortés, A., Margalef, T., Luque, E.: An Adaptive System for Forest Fire Behavior Prediction. In: 11th IEEE International Conference on Computational Science and Engineering (2008)

22. Iliadis, L.S.: A decision support system applying an integrated fuzzy model for long-term forest fire risk estimation. Environmental Modelling and Software 20, 613-621 (2005) 